\title{
Length-Biased Weighted Lomax Distribution: Statistical Properties and Application
}

\author{
Afaq Ahmad \\ Department of Statistics, University of Kashmir, Srinagar, India \\ baderaafaq@gmail.com \\ S.P Ahmad \\ Department of Statistics, University of Kashmir, Srinagar, India \\ spahmad@kashmiruniversity.net
}

\author{
A. Ahmed \\ Department of Statistics and Operation Research \\ Aligarh Muslim University, India \\ aqlstat@yahoo.co.in
}

\begin{abstract}
The concept of length-biased distribution can be employed in development of proper models for lifetime data. Length-biased distribution is a special case of the more general form known as weighted distribution. In this paper we introduce a new class of length-biased weighted Lomax distribution, (LBWLD). The statistical properties of this distribution are derived and the model parameters are estimated by maximum likelihood estimation and the observed information matrix is determined. An application to real data set is finally presented for illustration.
\end{abstract}

Keywords: Weighted distribution, Lomax distribution, Reliability Analysis, Maximum likelihood estimation, Order statistics, Real life data.

\section{Introduction}

The Lomax distribution also known as Pareto distribution of second kind has, in recent years, assumed opposition of importance in the field of life testing because of its uses to fit business failure data. Lomax distribution was introduced by Lomax (1974), Abdullah and Abdullah (2012) estimates the parameters of Lomax distribution based on generalized probability weighted moment. The Lomax distribution has been used in the literature in a number of ways. For example, it has been extensively used for reliability modeling and life testing; see, for example, Balkema and de Haan (1974). It also has been used as an alternative to the exponential distribution when the data are heavy tailed; see Bryson (1974). Ahsanullah (1991) studied the record values of Lomax distribution. Balakrishnan and Ahsanullah (1994) introduced some recurrence relations between the moments of record values from Lomax distribution. Also, the Lomax model has been studied, from a Bayesian point of view, by many authors; Nasiri and Hosseini (2012), Afaq et al. (2014) estimates the parameters of Lomax distribution using Jeffery's and extension of Jeffery's prior under different loss functions. The probability density function (pdf) of the Lomax distribution (LD) is given by

$$
f(x ; \theta, \lambda)=\frac{\theta}{\lambda}\left(1+\frac{x}{\lambda}\right)^{-(\theta+1)} \quad ; \quad x>0, \theta, \lambda>0
$$


Mean and variance is given by

$$
\begin{aligned}
& \mu_{1}^{\prime}=\frac{\lambda}{(\theta-1)} \\
& \mu_{2}=\frac{\lambda^{2} \theta}{(\theta-1)^{2}(\theta-2)}
\end{aligned}
$$

\section{Length-Biased Weighted Lomax Distribution}

Fisher (1934) introduced the concept of weighted distributions, Later it was introduced and formulated in general terms by Rao (1965), in connection with modeling statistical data where the usual practice of using standard distributions for the purpose was not found to be appropriate. In Rao's paper (1965), he identified various situations that can be modeled by weighted distributions. These situations refer to instances where the recorded observations cannot be considered as a random sample from the original distributions. This may occur due to non-observability of some events or damage caused to the original observation resulting in a reduced value or adoption of a sampling procedure which gives unequal chances to the units in the original. These distributions arise in practice when observations from a sample are recorded with unequal probability and provide unifying approach for the problems when the observations fall in the non experimental, non -replicated and non -random categories.

Suppose $X$ is a non-negative random variable with probability density function (pdf) $f(x)$, and then the pdf of the weighted random variable $X_{w}$ is given by

$$
f_{w}(x)=\frac{w(x) f(x)}{E(w(x))}, x>0
$$

where $w(x)$ be a non-negative weight function.

Depending upon the choice of the weight function $w(x)$, we have different weighted models. Clearly when $w(x)=x$, the resulting distribution is called length-biased whose pdf is given by:

$$
f_{l}(x)=\frac{x f(x)}{E(x)}, x>0
$$

Weighted distributions occur frequently in research related to reliability, bio-medicine, ecology and branching process can be seen in Gupta and Tripathi (1996), Patil and Rao (1986), Das and Roy (2011) discussed the length-biased Weighted Generalized Rayleigh distribution with its properties, also they develop the length-biased Weighted Weibull distribution.

A Length biased weighted Lomax distribution (LBWLD) is obtained by applying the weights $x^{c}$, where $\mathrm{c}=1$ to the weighted Lomax distribution. 
We have from relation equations (1) and (2)

$$
\begin{aligned}
& \mu_{1}^{\prime}=\int_{0}^{\infty} x f(x ; \theta, \lambda) d x=\frac{\lambda}{(\theta-1)} \\
& \int_{0}^{\infty} \frac{\theta(\theta-1)}{\lambda^{2}} x\left(1+\frac{x}{\lambda}\right)^{-(\theta+1)} d x=1
\end{aligned}
$$

This gives the Length biased weighted Lomax distribution as

$$
f_{l}(x ; \theta, \lambda)=\frac{\theta(\theta-1)}{\lambda^{2}} x\left(1+\frac{x}{\lambda}\right)^{-(\theta+1)} \quad ; \quad x>0, \theta, \lambda>0
$$

and the corresponding cdf is given by

$$
F_{l}(x ; \theta, \lambda)=1-\left(1+\frac{x}{\lambda}\right)^{-\theta}\left(1+\frac{x \theta}{\lambda}\right)
$$

where $\theta$ and $\lambda$ are shape and scale parameters, respectively.
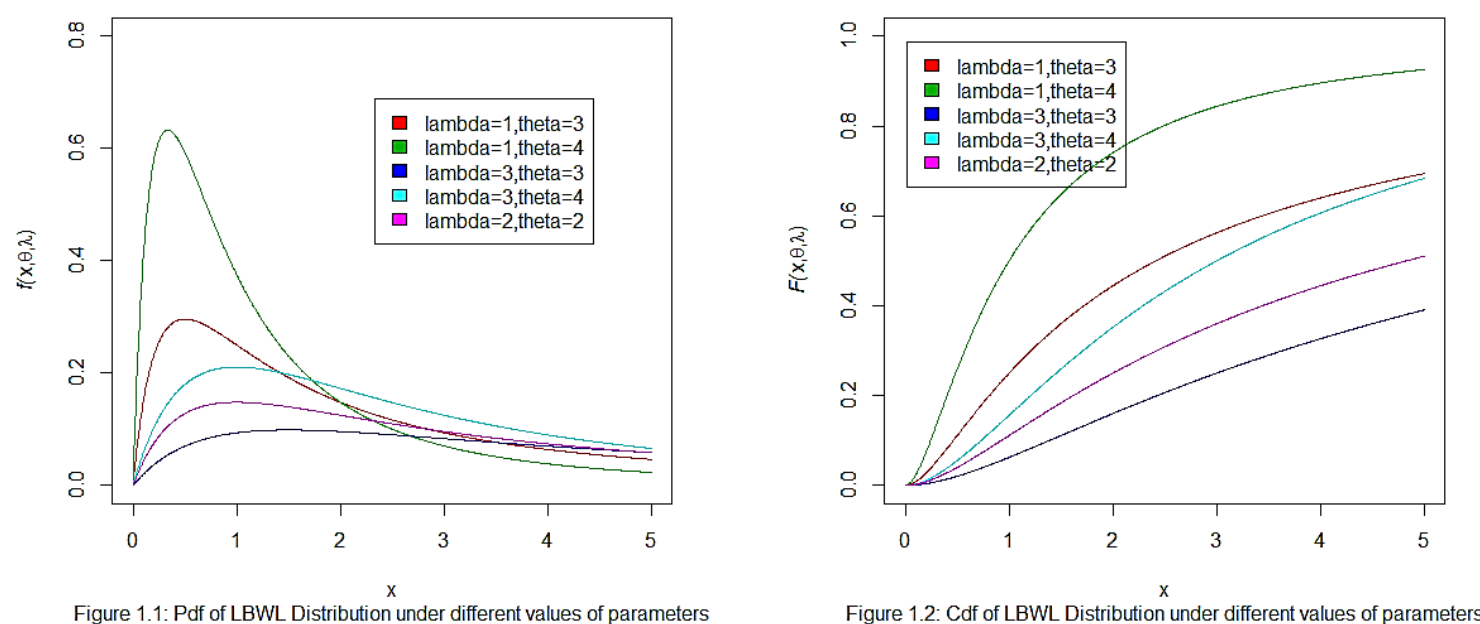

\section{Reliability Analysis}

In this sub-section, we present the reliability function and the hazard function for the proposed Length biased weighted Lomax distribution. The reliability function is otherwise known as the survival or survivor function. It is the probability that a system will survive beyond a specified time and it is obtained mathematically as the complement of the cumulative density function (cdf).

The survivor function is given by

$$
\begin{aligned}
& R(x)=1-F(x) \\
& R(x)=\left(1+\frac{x}{\lambda}\right)^{-\theta}\left(1+\frac{x \theta}{\lambda}\right)
\end{aligned}
$$


The hazard function is also known as the hazard rate, failure rate, or force of mortality and is given by

$$
\begin{aligned}
& h(x)=\frac{f(x)}{1-F(x)} \\
& h(x)=\frac{\theta(\theta-1)}{\lambda^{2}} x\left(1+\frac{x}{\lambda}\right)^{-1}\left(1+\frac{x \theta}{\lambda}\right)^{-1}
\end{aligned}
$$

The reverse hazard function for the Length biased weighted Lomax distribution is given as:

$$
\begin{aligned}
& h_{r}(x)=\frac{f(x)}{F(x)} \\
& h_{r}(x)=\frac{\frac{\theta(\theta-1)}{\lambda^{2}} x\left(1+\frac{x}{\lambda}\right)^{-(\theta+1)}}{1-\left(1+\frac{x}{\lambda}\right)^{-\theta}\left(1+\frac{x \theta}{\lambda}\right)}
\end{aligned}
$$

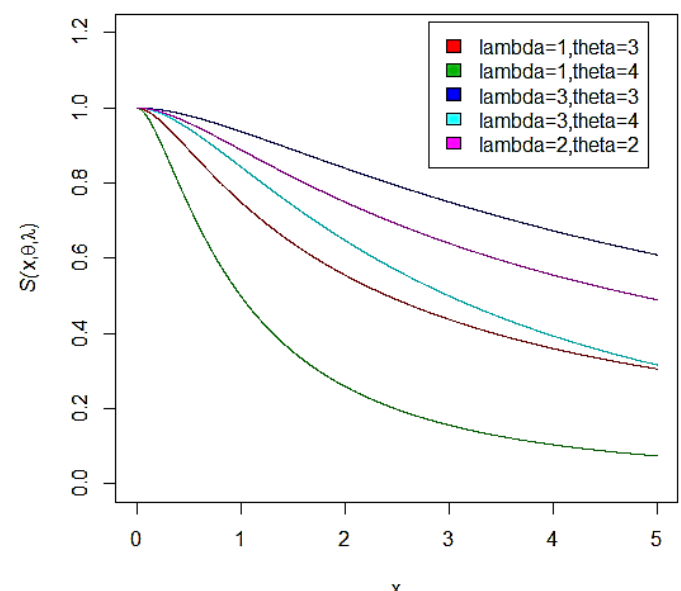

Figure 1.3: Survival function of LBWL Distribution under different values of paramı

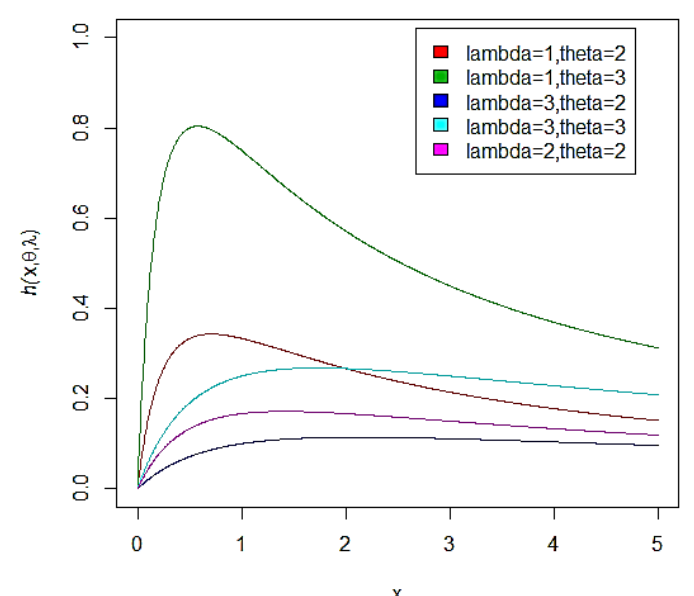

Figure 1.4: Failure rate of LBWL Distribution under different values to paramete

\section{Statistical Properties}

In this section we shall discuss structural properties of Length-Biased weighted Lomax distribution. Specially moments, order statistics, maximum likelihood estimation, moment generating function,

3.1 Moments: Suppose $X$ denote the Length-Biased weighted Lomax distribution random variable with parameters $\theta$ and $\lambda$, then

$$
E\left(X^{r}\right)=\mu_{r}^{\prime}=\int_{0}^{\infty} x^{r} f_{l}(x ; \theta, \lambda) d x
$$




$$
\begin{aligned}
& =\frac{\theta(\theta-1)}{\lambda^{2}} \int_{0}^{\infty} x^{r+1}\left(1+\frac{x}{\lambda}\right)^{-(\theta+1)} d x \\
& =\frac{\theta(\theta-1)}{\lambda^{2}} \int_{1}^{\infty} \lambda^{r+1}(t-1)^{r+1} t^{-(\theta+1)} \lambda d t \\
& =\theta(\theta-1) \lambda^{r} \int_{1}^{\infty}\left({ }^{r+1} C_{0} t^{r+1}-{ }^{r+1} C_{1} t^{r}+{ }^{r+1} C_{2} t^{r-1}-\ldots+{ }^{r+1} C_{r+1}(-1)^{r+1}\right) t^{-(\theta+1)} d t \\
& =\theta(\theta-1) \lambda^{r} \int_{1}^{\infty} \sum_{k=0}^{r+1}{ }^{r+1} C_{k} t^{r+1-k}(-1)^{k} t^{-(\theta+1)} d t \\
& =\theta(\theta-1) \lambda^{r} \sum_{k=0}^{r+1}(-1)^{k}{ }^{r+1} C_{k} \int_{1}^{\infty} t^{r-\theta-k} d t \\
& =\theta(\theta-1) \lambda^{r} \sum_{k=0}^{r+1}(-1)^{k}{ }^{r+1} C_{k}\left[\frac{t^{r-\theta-k+1}}{r-\theta-k+1}\right]_{1}^{\infty} \\
& \mu_{r}^{\prime}=\theta(\theta-1) \lambda^{r} \sum_{k=0}^{r+1}(-1)^{k+1} C_{k}^{r+1} C_{k}\left(\frac{1}{r-\theta-k+1}\right)
\end{aligned}
$$

Substitute $r=1,2,3,4$ we get first four moments

$$
\begin{aligned}
\text { Mean }= & \mu_{1}^{\prime}=\frac{2 \lambda}{(\theta-2)} \\
\mu_{2}^{\prime}= & \frac{6 \lambda^{2}}{(\theta-2)(\theta-3)}
\end{aligned}
$$

Variance $=\mu_{2}=\frac{2 \lambda^{2} \theta}{(\theta-2)^{2}(\theta-3)}$

Standard deviation $\sigma=\frac{\lambda}{(\theta-2)} \sqrt{\frac{2 \theta}{(\theta-3)}}$

Coefficient of variation $\quad C . V=\frac{\sigma}{\mu}=\frac{1}{2} \sqrt{\frac{2 \theta}{(\theta-3)}}$

\subsection{Moment generating function}

In this sub section we derived the moment generating function of LBWL distribution. We begin with the well known definition of the moment generating function given by

$$
M_{X}(t)=E\left(e^{t x}\right)=\int_{0}^{\infty} e^{t x} f_{l}(x ; \theta, \lambda) d x
$$




$$
\begin{aligned}
& =\int_{0}^{\infty}\left[1+t x+\frac{(t x)^{2}}{2 !}+\ldots\right] f_{l}(x ; \theta, \lambda) d x \\
& =\int_{0}^{\infty} \sum_{j=0}^{\infty} \frac{t^{j}}{j !} x^{j} f_{l}(x ; \theta, \lambda) d x \\
& =\sum_{j=0}^{\infty} \frac{t^{j}}{j !} \mu_{j}^{\prime} \\
& \Rightarrow M_{X}(t)=\theta(\theta-1) \sum_{j=0}^{\infty} \sum_{k=0}^{j+1}(-1)^{k+1} \frac{t^{j}}{j !} \lambda^{j}{ }^{j+1} C_{k}\left(\frac{1}{j-\theta-k+1}\right)
\end{aligned}
$$

\subsection{Mode}

In order to discuss monotonicity of LBWLD, we take the logarithm of its pdf as follows:

$$
\log f_{l}(x ; \theta, \lambda)=\log \left(\frac{\theta(\theta-1)}{\lambda^{2}}\right)+\log x-(\theta+1) \log \left(1+\frac{x}{\lambda}\right)
$$

Differentiating the above equation with respect to $x$ and equating to zero, we obtain

$$
x=\frac{\lambda}{\theta}
$$

\subsection{Order Statistics}

Order statistics make their appearance in many statistical theory and practice. We know that if $X_{(1)}, X_{(2)}, \ldots, X_{(n)}$ denotes the order statistics of a random sample $X_{1}, X_{2}, \ldots, X_{n}$ from a continuous population with $\operatorname{cdf} F_{X}(x)$ and pdf $f_{X}(x)$, then the pdf of $r$ th order statistics $X_{(r)}$ is given by

$$
f_{X(r)}(x)=\frac{n !}{(r-1) !(n-r) !} f_{X}(x)\left(F_{X}(x)\right)^{r-1}\left(1-F_{X}(x)\right)^{n-r}
$$

For $r=1,2, \ldots, \mathrm{n}$.

We have from (3) and (4) the pdf of the $r$ th order LBWL random variable $X_{(r)}$ is given by

$$
g_{X(r)}(x)=\frac{n !}{(r-1) !(n-r) !} \frac{\theta(\theta-1)}{\lambda^{2}} x\left(1+\frac{x}{\lambda}\right)^{-(\theta+1)}\left(\left(1+\frac{x}{\lambda}\right)^{-\theta}\left(1+\frac{x \theta}{\lambda}\right)\right)^{r-1}\left(1-\left(1+\frac{x}{\lambda}\right)^{-\theta}\left(1+\frac{x \theta}{\lambda}\right)\right)^{n-r}
$$

Therefore, the pdf of the nth order inverse Rayleigh statistic $X_{(\mathrm{n})}$ is given by

$$
g_{X(n)}(x)=\frac{n \theta(\theta-1)}{\lambda^{2}} x\left(1+\frac{x}{\lambda}\right)^{-(\theta+1)}\left(\left(1+\frac{x}{\lambda}\right)^{-\theta}\left(1+\frac{x \theta}{\lambda}\right)\right)^{n-1}
$$

and the pdf of the first order inverse Rayleigh statistic $X_{(1)}$ is given by

$$
g_{X(r)}(x)=\frac{n \theta(\theta-1)}{\lambda^{2}} x\left(1+\frac{x}{\lambda}\right)^{-(\theta+1)}\left(1-\left(1+\frac{x}{\lambda}\right)^{-\theta}\left(1+\frac{x \theta}{\lambda}\right)\right)^{n-1}
$$




\section{Estimation of parameters}

In this section, we discuss the various estimation methods for Length-Biased weighted Lomax distribution and verifying their efficiencies

\subsection{Methods of Moments}

Replacing sample moments with population moments, we get

$$
\begin{aligned}
& \frac{1}{n} \sum_{i=1}^{n} x_{i}=\mu_{1}^{\prime} \\
& \Rightarrow \bar{x}=\frac{2 \lambda}{\theta-2}
\end{aligned}
$$

and $\frac{1}{n} \sum_{i=1}^{n} x_{i}^{2}=\mu_{2}^{\prime}$

$$
\begin{aligned}
& \Rightarrow \frac{1}{n} \sum_{i=1}^{n} x_{i}{ }^{2}-\bar{x}^{2}=\mu_{2} \\
& \Rightarrow s^{2}=\frac{2 \lambda^{2} \theta}{(\theta-2)^{2}(\theta-3)} \\
& \Rightarrow \hat{\theta}=\frac{6 s^{2}}{2 s^{2}-\bar{x}^{2}}
\end{aligned}
$$

Substituting value of $\hat{\theta}$ in (13) we get estimate of $\lambda$ as given by

$$
\Rightarrow \hat{\lambda}=\bar{x}\left(\frac{6 s^{2}}{4 s^{2}-2 \bar{x}^{2}}-1\right)
$$

\subsection{Method of Maximum Likelihood estimator}

Maximum likelihood estimation has been the most widely used method for estimating the parameters of the Length biased Lomax distribution. Let $x_{1}, x_{2}, x_{3} \ldots \ldots . x_{n}$ be a random sample from the Length biased weighted Lomax distribution, then the corresponding likelihood function is given as

$$
L(X ; \theta, \lambda)=\frac{\theta^{n}(\theta-1)^{n}}{\lambda^{2 n}} \prod_{i=1}^{n} x_{i} \prod_{i=1}^{n}\left(1+\frac{x_{i}}{\lambda}\right)^{-(\theta+1)}
$$

The log-likelihood function is given as:

$\log L(x ; \theta, \lambda)=n \log \theta+n \log (\theta-1)-2 n \log \lambda+\sum_{i=1}^{n} \log x_{i}-(\theta+1) \sum_{i=1}^{n} \log \left(1+\frac{x_{i}}{\lambda}\right)$ 
Now, differentiate above equation with respect to parameters, we obtain the normal equations

$$
\begin{aligned}
& \frac{n(2 \theta-1)}{\theta(\theta-1)}=\sum_{i=1}^{n} \log \left(1+\frac{x_{i}}{\lambda}\right) \\
& \frac{2 n}{\lambda}=\frac{(\theta+1)}{\lambda^{2}} \sum_{i=1}^{n} \frac{x_{i}}{\left(1+\frac{x_{i}}{\lambda}\right)}
\end{aligned}
$$

The MLE $\hat{\eta}=(\hat{\theta}, \hat{\lambda})$ of $\eta=(\theta, \lambda)$ is obtained by solving this nonlinear system of equations. It is usually more convenient to use nonlinear optimization algorithms such as quasi-Newton algorithm to numerically maximize the log likelihood function given in (16). Applying the usual large sample approximation, the MLE $\hat{\eta}$ can be treated as being approximately bivariate normal with variance-covariance matrix equal to the inverse of the expected information matrix, i.e.

$$
\sqrt{n}(\hat{\eta}-\eta) \rightarrow N\left(0, n I^{-1}(\eta)\right)
$$

where $I^{-1}(\eta)$ is the limiting variance-covariance matrix of $\hat{\eta}$.

The elements of $2 \times 2$ Fisher Information matrix is given below

$$
\begin{aligned}
& I(\theta, \lambda)=\left[\begin{array}{l}
-E\left(\frac{\partial^{2}}{\partial \theta^{2}} \log f_{l}(x ; \theta, \lambda)\right)-E\left(\frac{\partial^{2}}{\partial \theta \partial \lambda} \log f_{l}(x ; \theta, \lambda)\right) \\
-E\left(\frac{\partial^{2}}{\partial \lambda \partial \theta} \log f_{l}(x ; \theta, \lambda)\right)-E\left(\frac{\partial^{2}}{\partial \lambda^{2}} \log f_{l}(x ; \theta, \lambda)\right)
\end{array}\right] \\
&= {\left[\begin{array}{ll}
I_{11} & I_{12} \\
I_{21} & I_{22}
\end{array}\right] } \\
& I_{11}=-E\left(\frac{\partial^{2}}{\partial \theta^{2}} \log L(x ; \theta, \lambda)\right)=\frac{2 \theta^{2}-2 \theta+1}{\theta^{2}(\theta-1)^{2}} \\
& I_{12}=I_{21}=-E\left(\frac{\partial^{2}}{\partial \theta \partial \lambda} \log L(x ; \theta, \lambda)\right)=\frac{-2(\theta-2)}{\theta(\theta-1)} \\
& I_{22}=E\left(\frac{\partial^{2}}{\partial \lambda^{2}} \log L(x ; \theta, \lambda)\right)=-\frac{2}{\lambda^{2}}+\frac{(\theta+1)}{\lambda^{2}} \frac{(4 \theta+2)}{\theta(\theta-1)}
\end{aligned}
$$

\section{Test for Length-biasedness of Length biased weighted Lomax distribution}

Let $x_{1}, x_{2}, x_{3} \ldots \ldots x_{n}$ be a random sample from the Lomax distribution or Length biased weighted Lomax distribution. We test the hypothesis

$$
H_{o}: f(x)=f(x ; \theta, \lambda) \text { against } H_{1}: f(x)=f_{l}(x ; \theta, \lambda)
$$


For testing whether the random sample of size $\mathrm{n}$ comes from the Lomax distribution or Length biased weighted Lomax distribution, the following test statistic is used

$$
\begin{aligned}
& \Delta=\frac{L_{1}}{L_{o}}=\prod_{i=1}^{n} \frac{f_{l}(x ; \theta, \lambda)}{f(x ; \theta, \lambda)} \\
& \Delta=\frac{L_{1}}{L_{o}}=\prod_{i=1}^{n}\left[\frac{\frac{\theta(\theta-1)}{\lambda^{2}} x_{i}\left(1+\frac{x_{i}}{\lambda}\right)^{-(\theta+1)}}{\frac{\theta}{\lambda}\left(1+\frac{x_{i}}{\lambda}\right)^{-(\theta+1)}}\right] \\
& \Delta=\frac{L_{1}}{L_{o}}=\prod_{i=1}^{n}\left[\frac{(\theta-1)}{\lambda} x_{i}\right] \\
& \Delta=\frac{L_{1}}{L_{o}}=\left(\frac{(\theta-1)}{\lambda}\right)^{n} \prod_{i=1}^{n} x_{i}
\end{aligned}
$$

We reject the null hypothesis if

$$
\frac{(\theta-1)}{\lambda} \prod_{i=1}^{n} x_{i}>k
$$

Equalivalently, we reject the null hypothesis where

$$
\Delta^{*}=\prod_{i=1}^{n} x_{i}>k^{*}, \text { where } k^{*}=k\left(\frac{(\theta-1)}{\lambda}\right)^{n}>0
$$

For a large sample of size $n, 2 \log \Delta$ is distributed as chi-square distribution with one degree of freedom. Thus p-value is obtained from the chi-square distribution. Also we reject the null hypothesis, when probability value is given by:

$P\left(\Delta^{*}>\alpha^{*}\right)$, where $\alpha^{*}=\prod_{i=1}^{n} x_{i}$ is less than a specified level of significance, where $\prod_{i=1}^{n} x_{i}$ is the observed value of the statistic $\Delta^{*}$.

\section{Application}

In this section, we use a real data set to show that the Length biased Lomax distribution can be a better model than the Lomax distribution. We consider a data set corresponding to remission times (in months) of a random sample of 128 bladder cancer patients given in Lee and Wang (2003). The data set is given as follows : 0.08, 2.09, 2.73, 3.48, 4.87, $6.94,8.66,13.11,23.63,0.20,2.22,3.52,4.98,6.99,9.02,13.29,0.40,2.26,3.57,5.06$, $7.09,9.22,13.80,25.74,0.50,2.46,3.64,5.09,7.26,9.47,14.24,25.82,0.51,2.54,3.70$, $5.17,7.28,9.74,14.76,26.31,0.81,2.62,3.82,5.32,7.32,10.06,14.77,32.15,2.64,3.88$, $5.32,7.39,10.34,14.83,34.26,0.90,2.69,4.18,5.34,7.59,10.66,15.96,36.66,1.05$, $2.69,4.23,5.41,7.62,10.75,15.62,43.01,1.19,2.75,4.26,5.41,7.63,17.12,46.12,1.26$, $2.83,4.33,5.49,7.66,11.25,17.14,79.05,1.35,2.87,5.62,7.87,11.64,17.36,1.40,3.02$, 
$4.34,5.71,7.93,11.79,18.10,1.46,4.40,5.85,8.26,11.98,19.13,1.76,3.25,4.50,6.25$, $8.37,12.02,2.02,3.31,4.51,6.54,8.53,12.03,20.28,2.02,3.36,6.93,8.65,12.63$ and 22.69. These data are used here only for illustrative purposes. The required numerical evaluations are carried out using the Package of R software.

We have fitted Lomax and Length biased weighted Lomax models to this data. These two distributions are fitted to the subject data using maximum likelihood estimation. The MLEs of the parameters with standard errors in parentheses and the corresponding loglikelihood values, AIC, AICC and BIC are displayed in Table 1.

Table 1: MLEs (S.E in parentheses) and Criteria for Comparison

\begin{tabular}{|l|l|l|l|l|l|l|}
\hline Distribution & $\theta$ & \multicolumn{1}{|c|}{$\lambda$} & $-2 \log L$ & AIC & AICC & BIC \\
\hline Lomax & $\begin{array}{l}11.091 \\
(9.292)\end{array}$ & $\begin{array}{l}94.472 \\
(85.696)\end{array}$ & 795.125 & 799.125 & 799.221 & 799.339 \\
\hline $\begin{array}{l}\text { Length biased } \\
\text { Lomax }\end{array}$ & $\begin{array}{l}4.325 \\
(0.930)\end{array}$ & $\begin{array}{l}10.974 \\
(3.782)\end{array}$ & 789.74 & 793.74 & 793.836 & 793.954 \\
\hline
\end{tabular}

In order to compare the two distribution models, we consider the criteria like AIC (Akaike information criterion), AICC (corrected Akaike information criterion) and BIC (Bayesian information criterion). The better distribution corresponds to lesser AIC, AICC and BIC values.

$$
\mathrm{AIC}=2 \mathrm{k}-2 \log L \quad \mathrm{AICC}=\mathrm{AIC}+\frac{2 k(k+1)}{n-k-1} \text { and } \mathrm{BIC}=\mathrm{k} \log n-2 \log L
$$

where $\mathrm{k}$ is the number of parameters in the statistical model, $\mathrm{n}$ is the sample size and $2 \log L$ is the maximized value of the $\log$-likelihood function under the considered model. From Table 1, it has been observed that the Length biased Lomax distribution have the lesser AIC, AICC and BIC values as compared to Lomax Distribution. Hence we can concluded that the Length biased Lomax distribution leads to a better fit than the Lomax distribution

\section{Conclusion}

This article defined a Length biased Lomax distribution (LBWLD) and studied various properties of the distribution. The moments, mode, survival function, hazard function and the maximum likelihood estimates of the parameters, have been investigated. The application of the new distribution has also been demonstrated with real life data. The results, are compared with Lomax distribution, revealed that the LBWLD provides a better fit than the Lomax distribution.

\section{Acknowledge}

The Authors appreciate the efforts of the anonymous referees for their timely comments towards improving the quality of this article. 


\section{References}

1. Abdullah, M.A. and Abdullah, H.A., (2010). Estimation of Lomax parameters based on Generalized Probability Weighted Moment, JKAU, Sci (22), pp. 171-184.

2. Afaq, A., Ahmad S.P., and Ahmed A., (2010). Bayesian Analysis of shape parameter of Lomax distribution under different loss functions, International Journal of Statistics and Mathematics, vol. 2 (1), pp. 055-065.

3. Ahsanullah, M., (1991). Record values of Lomax distribution, Statistica Nederlandica, vol. 41, no. 1, pp. 21-29.

4. Balakrishnan, N and Ahsanullah, M., (1994). Relations for single and product moments of record values from Lomax distribution, Sankhya B, vol. 56, no. 2, pp. $140-146$.

5. Balkema, A. and L. de Haan, (1974). Residual life time at great age, Annals of Probability, vol. 2, no. 5, pp. 792-804.

6. Bryson, M.C., (1974). Heavy-tailed distributions: properties and tests, Technometrics, vol. 16, no. 1, pp. 61-68.

7. Das K. K. and Roy T. D. (2011). On Some Length-Biased Weighted Weibull Distribution, Pelagia Research Library, Advances in Applied Science Research, vol.2 (5), pp.465-475.

8. Das, K. K. and Roy, T. D. (2011). Applicability of Length Biased Weighted Generalized Rayleigh distribution, Advances in Applied Science Research, 2 (4), pp.320-327.

9. Fisher, R.A (1934). The effects of methods of ascertainment upon the estimation of frequencies. Ann. Eugenics, 6, 13-25.

10. Lomax, H.S., (1954). Business Failures, Another example of the analysis of Failure data, JASA, (49), pp.847-852.

11. Nasiri, P. and Hosseini, S., (2012). Statistical Inferences for Lomax distribution based on record values (Bayesian and Classical), Journal of Modern Applied Statistical Methods, vol.11, pp. 179-189.

12. Patill, G.P. and Rao, C.R. and Ratnaparkhi, M.V. (1986). On discrete weighted distribution and their use in model choice for observed data. Commun. StatisitTheory Math, 15(3), 907-918.

13. Rao, C.R. (1965). On discrete distributions arising out of method of ascertainment, in classical and Contagious Discrete, G.P. Patil. ed; Pergamum Press and Statistical publishing Society, Calcutta, pp-320-332.

14. Lee, E.T. and Wang, J.W. (2003). Statistical Methods for Survival Data Analysis, 3 edn, John Willey, New York. 